\title{
EL VÍNCULO INTERACTIVO BIBLIOTECA UNIVERSITARIA-USUARIO EN EL SIGLO XXI: ALGUNAS CONSIDERACIONES PARA FORTALECER ESTE RELACIONAMIENTO.
}

\author{
THE INTERACTIVE RELATIONSHIP BETWEEN THE UNIVERSITY LIBRARY AND \\ THE USERS IN THE XXITH CENTURY: SOME CONSIDERATIONS TO STRENGTHEN \\ THESE CONNECTION.
}

Lic. MARÍA GLADYS CERETTA SORIA

Docente de la Escuela Universitaria de Bibliotecología Universidad de la República, URUGUAY

E-mail: eubca@adinet.com.uy

\section{RESUMEN}

El relacionamiento entre la biblioteca universitaria y la heterogenidad de sus usuarios (estudiantes, docentes, investigadores, graduados), ha sufrido grandes transformaciones como consecuencia del impacto provocado por las nuevas formas de comunicación y de acceso a la información, producto de los dinámicos avances tecnológicos. Frente a las tendencias que pronostican que la biblioteca universitaria ha dejado de tener sentido en un mundo en el que la información supera los límites físicos de una institución y en el cual el usuario se relaciona directa e individualmente con la información a través de la computadora, se promueve el rol fundamental de la biblioteca universitaria como mediadora de los procesos de enseñanzaaprendizaje y de apoyo a la investigación y a la generación de nuevo conocimiento. Se considera que una de las formas apropiadas para favorecer este rol es establecer canales de comunicación que fortalezcan el vínculo interactivo biblioteca-usuario a través de la consolidación institucional de instancias de orientación y formación de usuarios, destacándose el papel fundamental que en este proceso de relacionamiento desarrolla el profesional Bibliotecólogo. Se toma como punto de referencia la situación actual que al respecto presentan las Bibliotecas Universitarias en el Uruguay. Se establecen algunas pautas generales para el desarrollo de esta propuesta.

Palabras clave: bibliotecas universitarias; usuário; Uruguay

\section{INTRODUCCION}

Las bibliotecas universitarias se han concebido tradicionalmente como grandes centros bibliográficos de apoyo académico especializado a los más variados intereses temáticos que convergen en ellas, provenientes de la diversidad de usuarios que le son propios: estudiantes, docentes, graduados, investigadores. Esta particularidad ha enfrentado a la biblioteca universitaria a contemplar desde su gestión respuestas a las distintas necesidades informativas y comportamientos de sus usuarios, situación que se ha visto reflejada en sus colecciones, 
servicios y en la búsqueda de distintas estrategias de relacionamiento y comunicación con su heterogénea comunidad.

Los dinámicos avances en las tecnologías de la comunicación y de la información han aparejado nuevas formas de relacionamiento entre el usuario y la biblioteca que han impactado fuertemente en las distintas unidades de información y en forma muy particular en las bibliotecas universitarias.

"Ha cambiado el rol de la información y está cambiando la situación relativa a las unidades de información : hoy se habla de bibliotecas sin paredes, bibliotecas virtuales y bibliotecas digitales" (1) y esta situación no es ajena al contexto en el que se desarrollan las bibliotecas universitarias e influye directamente en la relación biblioteca-usuario.

Es hacía mediados del siglo XX cuando comienzan a producirse los primeros grandes cambios en las formas de relacionamiento biblioteca-usuario. Lentamente, el usuario se va convirtiendo en el centro de atención de las distintas unidades de información, y comienza a interactuar con las mismas.

La biblioteca pasa de ser un depósito de conservación de materiales a ser un centro dinámino, preocupado, comprometido, planificado en virtud del uso real o potencial que de ella puedan hacer los interesados.

Es a partir de este momento que el relacionamiento biblioteca- usuario comienza a visualizarse desde enfoques sicológicos y sociológicos, enfatizándose especialmente en la necesidad de crear entre el usuario y la biblioteca el contexto apropiado para generar entre ambos un vínculo de relacionamiento interactivo.

En qué consiste este vínculo de relacionamiento interactivo?. En esencia supone un proceso de comunicación entre el usuario y la biblioteca, que implica para ambas partes instancias de acercamiento y mutuo conocimiento, en donde se destaca la presencia fundamental del profesional Bibliotecólogo cumpliendo funciones de nexo o intermediario entre el usuario y la biblioteca.

Este vínculo de relacionamiento interactivo biblioteca-usuario es indispensable para el gerenciamiento de las unidades de información, ya que a través del mismo se obtienen los insumos necesarios para implementar servicios acordes a la comunidad de usuarios a la que se sirve.

Pero, los avances tecnológicos han cambiado radicalmente la forma en que los usuarios se comunican y usan los recursos de información (2). Esto se puede apreciar concretamente en 
su relación con la biblioteca y en la generación de un usuario con características y comportamientos nuevos.

Y así, nos vemos enfrentados a un usuario que cada día dispone de menos tiempo para consultar la biblioteca y generalmente cuando lo hace es porque busca una información puntual. Es frecuente que nos comuniquemos con él a través de los medios electrónicos y nunca lleguemos a conocerlo personalmente. Un usuario que se comporta con mayor individualismo, autonomía y autosuficiencia ya que tiene la posibilidad de acceder a la información sin intermediarios, desde su propia casa, desde su trabajo, desde la biblioteca, o desde el lugar en donde se encuentre siempre que tenga un computador disponible. Está cerca del mundo pero lejos de quienes lo rodean. Mantiene una actitud interactiva con la computadora que le brinda la posibilidad de conectarse con el mundo a través de INTERNET, o comunicarse por correo electrónico. Aparentemente no hay límites físicos para acceder a la información. También es común que exista en el usuario la creencia de que la posibilidad de acceder a los medios electrónicos ha sustituído el rol de la biblioteca.

Pero la realidad nos dice que los medios electrónicos son herramientas de gran utilidad para el manejo de la información, pero que es necesario que el usuario se enfrente a los mismos con criterios de evaluación y selección de la información en ellos contenida para no verse superado por un caudal informativo incontrolable.

En este contexto que se generaliza rápidamente, ¿cómo enfrentar desde la biblioteca estos nuevos comportamientos del usuario?. ¿cómo revalorizar el rol de la biblioteca universitaria y del profesional Bibliotecólogo?.

Creemos que las herramientas proporcionadas por la tecnología son elementos facilitadores en el proceso de búsqueda y recuperación de la información, pero que no sustituyen el rol fundamental que en el medio académico desempeñan las bibliotecas universitarias como mediadoras de los procesos de enseñanza-aprendizaje y de apoyo a la investigación y a la generación de nuevo conocimiento.

Hoy la biblioteca universitaria enfrenta nuevos desafíos que pasan no solo por estar al día con las últimas tecnologías incorporándolas al servicio, sino y muy especialmente por la búsqueda de nuevas formas de comunicación y relacionamiento que fortalezcan el vínculo interactivo con su comunidad de usuarios.

En este sentido, la presente ponencia se propone fundamentar en líneas generales, que una de las posibles formas para fortalecer el vínculo interactivo biblioteca universitaria-usuario, 
en este nuevo mundo tecnológico, es la implementación de acciones institucionales orientadas hacia las estrategias de formación de los usuarios para uso de la información.

\section{LAS BIBLIOTECAS UNIVERSITARIAS EN EL URUGUAY}

Parece oportuno conocer brevemente un panorama de la realidad de las Bibliotecas Universitarias en el Uruguay considerando aspectos generales y enfatizando especialmente en la relación biblioteca-usuario para promover la implementación de instancias de formación de usuarios para el uso de la información.

A modo de ubicarnos en la temática, digamos que Uruguay, es un país que cuenta con una superficie de 187.000 kilómetros, y aproximadamente una población de tres millones de habitantes, situado al sur de América Latina, delimitado por Brasil, Argentina, el Río de la Plata y el Océano Atlántico. Es miembro fundador del MERCOSUR junto con sus socios Brasil, Argentina y Paraguay y en Montevideo se ubica la sede capital de este emprendimiento regional.

Con respecto a la educación universitaria en el Uruguay, la misma tiene su principal centro en la Universidad de la República, institución de carácter público creada hace más de 150 años, a pesar de que en los últimos años se han creado dos o tres universidades privadas.

De acuerdo al Censo de Estudiantes realizado en noviembre de 1999 la Universidad de la República cuenta con un total de 66.500 estudiantes y como dato significativo es a través de ella que se genera prácticamente el $90 \%$ de la investigación que se realiza en el país.

Es en la órbita de la Universidad de la República que funcionan más de 35 bibliotecas universitarias que abarcan todas las disciplinas, algunas de ellas únicas en el país (ejemplo Bibliotecología). Prácticamente el total de estas bibliotecas se ubican geográficamente en la ciudad de Montevideo a distancias relativamente pequeñas unas de las otras.

Las bibliotecas universitarias están organizadas en base a un modelo descentralizado en el cual cada Facultad, Instituto o Escuela tiene su biblioteca y/o centro de documentación que contempla sus propios intereses temáticos y apoya el desarrollo de la enseñanza, la docencia y la investigación en su área.

Algunas de estas bibliotecas han alcanzado una importancia muy significativa en el medio académico, como por ejemplo: a) la Biblioteca de la Facultad de Medicina, instituída Biblioteca Nacional de Medicina (BINAME), sede de Centro Nacional de Información en Medicina y Ciencias de la Salud (CENDIM,) coordinadora de la Red de Bibliotecas 
Biomédicas en el Uruguay y Centro de la Biblioteca Virtual en Salud. La Biblioteca Nacional de Medicina es integrante de redes a nivel regional e internacional como ser BIREME y National Library of Medicine; b) la Biblioteca de la Facultad de Derecho, cuya creación se remonta prácticamente a los inicios de la Universidad (150 años), que cuenta con una colección de más de 400.000 volúmenes integrada por materiales únicos en el país de gran valor histórico y temático; c) la Biblioteca de la Facultad de Ciencias, que a pesar de haber sido creada en esta última década, ha logrado desarrollar un colección altamente especializada y de punta en el área científico-tecnológica.

Hasta el momento, la Universidad de la República no ha establecido políticas generales para el desarrollo conjunto de las bibliotecas universitarias y a pesar de no existir un vínculo formal de relacionamiento entre las bibliotecas universitarias y tampoco contar con la presencia de un Coordinador de Bibliotecas, que actúe de nexo comunicador entre ellas, de igual forma y generalmente por iniciativa de las propias bibliotecas se ha promovido la realización de acciones conjuntas de cooperación e intercambio entre ellas, encontrando los canales de comunicación apropiados que han beneficiado el funcionamiento de todos los servicios, con la finalidad de racionalizar y aprovechar al máximo los recursos existentes.

En este sentido son sumamente destacables algunos ejemplos de este tipo de iniciativas: a) el "Catálogo Colectivo de Publicaciones Periódicas Existentes en las Bibliotecas Universitarias del Uruguay", al cual se puede acceder en forma automatizada, y su base de datos está volcada a INTERNET. Este catálogo permite la localización física de existencias de títulos de publicaciones periódicas en las distintas bibliotecas universitarias; b) el préstamo interbibliotecario por el cual el usuario de la biblioteca universitaria puede acceder al préstamo de publicaciones existentes en todas las bibliotecas universitarias; c) intentos de integración de un Sistema de Información Bibliográfica de la Universidad de la República (SIBUR) a través de una Red de Información Bibliográfica; d) emprendimientos relacionados con la centralización en la selección y adquisición de colecciones con miras a racionalizar recursos económicos e informativos.

Asimismo, las bibliotecas universitarias no han permanecido al margen de los avances tecnológicos. En este sentido, podemos decir que a pesar de la desigualdad en los equipamientos informáticos, la mayoría tiene su propia página WEB; han volcado sus bases de datos en INTERNET, lo que posibilita la consulta de las mismas desde cualquier lugar del mundo; desde ellas se puede consultar bases de datos en línea; utilizan correo electrónico; e incluso algunas de ellas intentan convertirse en verdaderas bibliotecas virtuales. 
Con respecto a sus colecciones, están integradas por libros y especialmente título de publicaciones periódicas en soporte manual y electrónico. La actualización de las colecciones de publicaciones periódicas es una problemática candente a causa de la limitación en los recursos financieros, lo que ocasiona grandes lagunas en las colecciones con las consiguientes dificultades que ello ocasiona.

Generalmente, las bibliotecas universitarias brindan servicios de préstamo en sala y a domicilio; préstamo interbibliotecario; referencia; búsquedas bibliográficas automatizadas y en línea; diseminación general y selectiva, extensión y formación de usuarios.

Respecto a actividades relacionadas con la formación de usuarios podríamos decir que es una actividad que formal o informalmente está presente en todas las bibliotecas universitarias, pero son especialmente destacables los esfuerzos que al respecto realizan algunas bibliotecas que cuentan con programas estables de formación de usuarios, dirigidos principalmente a estudiantes, como por ejemplo el Instituto de Enfermería, la Facultad de Odontología, la Facultad de Derecho y la Facultad de Medicina.

Ahora bien, en este panorama general ¿cómo es la relación biblioteca universitaria-usuario en el Uruguay?

Podría decirse que se dan distintas modalidades de relacionamiento desde el tradicional pasando por formas intermedias, hasta el virtual según el entorno con el que cuenta la biblioteca y con el tipo de usuario con el que se relaciona.

Pero, para responder a esta pregunta se hace necesario visualizar el tema de la relación biblioteca-usuario, considerando el mismo brevemente desde la diversidad de usuarios (estudiantes, docentes, graduados, investigadores) que hacen uso de la biblioteca universitaria, sus diferentes universos de necesidades y comportamientos y sus problemáticas particulares.

En tal sentido y a los efectos de sistematizar el tema se enfocaremos el mismo desde las siguientes categorías:

\section{A) Relación biblioteca universitaria - estudiantes:}

La relación biblioteca universitaria-estudiantes presenta las siguientes particularidades:

a) la presencia del fenómeno de la masificación al que se enfrenta hoy la Universidad de la República y en general las universidades latinoamericanas que se produce por el ingreso masivo de estudiantes a la Universidad (por ejemplo este año han ingresado a la Facultad de Ciencias Económicas más de 2500 estudiantes, y casi similar la cifra que ingresó a Facultad de Derecho), es un factor que se refleja directamente en el número de personas 
que son potenciales usuarios de la biblioteca universitaria, así por ejemplo hay bibliotecas que pueden atender más de mil usuarios por día.

1. El excesivo número de usuarios es una determinante que impone formas de relacionamiento y comunicación entre la biblioteca y el usuario que no siempre son las deseables para propiciar un vínculo interactivo entre ambos. Esto conlleva la dificultad para instrumentar servicios al público y muchas veces condena a la unidad de información a ser en un mero recinto de almacenaje y despacho de publicaciones.

b) generalmente cuando el estudiante ingresa a la Universidad no ha recibido en sus instancias educativas anteriores una capacitación específica para saber manejarse con la información y es en ese momento que motivado por las propias exigencias académicas comienza a valorar la importancia de saber hacer uso de la misma. Asimismo, se debe tener en cuenta que las necesidades informativas y los comportamientos de los usuarios estudiantes universitarios en relación al uso y manejo de la información van cambiando a medida que transcurren los años de formación universitaria.

\section{B) Relación biblioteca universitaria-docente/investigador}

A pesar que no necesariamente las calidades de docente e investigador se tienen que dar en forma conjunta en una misma persona, generalmente en nuestra Universidad la docencia y la investigación están muy ligadas.

Este tipo de usuario que es un especialista y conocedor en profundidad del área temática de su interés generalmente se comporta en su relación con la biblioteca de la siguiente forma:

a) ha aprendido a interactuar con la biblioteca es exigente en sus requerimientos y muchas veces un verdadero experto en manejo de la información.

b) generalmente tiene la posibilidad de acceder a la información a través de medios tecnológicos.

c) se comunica con sus pares a través del correo electrónico, dándole una dinámica diferente a la consolidación de los colegios invisibles.

d) Espera encontrar en la biblioteca un profesional Bibliotecólogo altamente especializado con el cual pueda comunicarse de igual a igual.

\section{C) Relación biblioteca universitaria-graduados}

En esta categoría de relación podemos encontrar diferentes comportamientos que pueden ir desde el usuario que recurre a la biblioteca para ser orientado y asesorado hasta el que es 
realmente un experto en el manejo de información. Generalmente sus necesidades informativas están pautadas por su desempeño desde el punto de vista profesional y apuntan a la resolución de situaciones concretas.

Como podemos observar, cada categoría planteada da lugar a una relación biblioteca-usuario enmarcada por características, comportamientos y necesidades diferentes a las que la biblioteca universitaria debe hacer frente.

En este sentido, las bibliotecas universitarias han sabido sortear esta gran dificultad y se han orientado a la búsqueda de diferentes canales de comunicación ya sean formales o informales que construyan un relacionamiento efectivo con sus usuarios. Así por ejemplo: a) desde la implementación de servicios como los de préstamo o de información y consulta (referencia) se propicia el relacionamiento directo biblioteca-usuario utilizando como nexo la funciones de orientación y asesoramiento inherentes a la profesión de Bibliotecólogo ; b) se han creado Comisiones de Biblioteca, como nexo entre el servicio y las necesidades de los usuarios, integradas generalmente por usuarios representantes de su comunidad, algunos de ellos altamente calificados; c) se llevan adelante estudios de usuarios, como punto de partida para la planificación, evaluación y toma de decisiones; d) se implementan programas formales de formación de usuarios en el uso de la información. (3)

En este sentido, es de destacar que las bibliotecas universitarias han sido tradicionalmente conscientes de la importancia que apareja el buscar formas apropiadas para fortalecer efectivamente la relación interactiva biblioteca-usuario.

\section{LA FORMACIÓN DE USUARIOS y EL ROL DEL BIBLIOTECÓLOGO}

En principio y solamente a modo de introducción, diremos que es un tema sobre el cual aún no se ha profundizado en fundamentos teóricos y que generalmente es producto de experiencias prácticas llevadas a cabo en marcos institucionales, por iniciativa de los propios profesionales Bibliotecólogos.

Es así, que aún no se ha acordado la terminología más adecuada para conceptualizar esta actividad bibliotecológica y se han utilizado para denominarlo en forma indistinta términos tales como: formar, educar, adiestrar, instruir, orientar, capacitar.

Así por ejemplo la UNESCO (4) ha definido la educación y la formación del usuario en una forma genérica como "todo proyecto o programa destinado a orientar e instruir a los usuarios 
actuales y potenciales, individual o colectivamente, con el objetivo de facilitar: a) el reconocimiento de sus propias necesidades de información; b) la formulación de estas necesidades; c) la utilización efectiva y eficaz de los servicios de información, así como; d) la evaluación de estos servicios".

Por su parte Hernández (5) habla de formación de usuarios en uso de información y la define como "el proceso de intercambio de experiencias o saberes significativos sobre el uso de la información, con el fin de que la persona que la usa, de acuerdo con su proceso cognoscitivo pueda cambiar su actitud y aptitud en forma positiva, optimando dicha utilización al máximo".

No es objetivo de este trabajo profundizar en el estudio de cual de los términos mencionados es el más adecuado para denominar la formación de usuarios para el uso de la información, sí nos interesa rescatar la esencia del concepto, que implica una instancia de relacionamiento biblioteca-usuario que tiene como intermediario la presencia protagónica del profesional Bibliotecólogo y que apunta a brindar al usuario elementos significativos que le permitan evaluar la información y hacer uso de la misma aprovechando al máximo los recursos informativos a los que tiene acceso.

La formación de usuarios implica un acercamiento real con el usuario y a través de la misma se puede conocer desde la biblioteca con mayor exactitud sus necesidades informativas y comportamientos y especialmente propiciar un alto grado de interacción entre ambos.(6)

Al decir de la Profa. Saray Córdoba (7) "la formación de usuarios es una tarea que generalmente se mantiene relegada en las unidades de información por razones de ahorro de tiempo, recursos económicos o humanos o porque simplemente es una actividad cuyos resultados no se observan a corto plazo...."

Entonces, ¿para que implementar instancias de formación de usuarios a nivel de las bibliotecas universitarias?

A pesar de no ser una situación totalmente generalizada en nuestro medio, la biblioteca universitaria se enfrenta hoy a un usuario que bajo la influencia de los beneficios de las nuevas tecnologías de la comunicación y la información se ve expuesto a un universo inabarcable de información por lo que creemos que es a través de estas instancias de formación que lograremos:

a) Promover la capacitación de un usuario consciente, crítico y selectivo de la información a la que tiene acceso tanto en forma manual como a través de los nuevos medios tecnológicos. 
b) Brindar al usuario los elementos necesarios para que sea capaz de adquirir, entender, transformar y difundir la información generando nuevos conocimientos.

Por lo tanto, cuando nos referimos a la formación de usuarios para el uso de la información a nivel universitario, debemos pensar en una actividad orientada a la generación de usuarios que se enfrenten a la información con una actitud crítica, aptos para distinguir la credibilidad de sus fuentes y saber evaluar y seleccionar la misma.

Las actividades de formación de usuarios en las bibliotecas universitarias deberán contemplar estrategias diversas que tengan en cuenta las diferentes modalidades de relacionamiento, comportamientos y necesidades informativas de estudiantes, docentes, investigadores y graduados.

Al mismo tiempo, podrán implementarse: a) instancias formales a través de la planificación, evaluación y seguimiento de programas específicos de formación de usuarios; b) instancias informales a través de la orientación que brinde el profesional Bibliotecólogo en su interacción directa con el usuario, como una actividad inherente a su profesión.

El rol del profesional Bibliotecólogo en la formación de usuarios es imprescindible y su importancia fundamental radica en que es un especialista altamente capacitado en la evaluación y uso de la información y está en inmejorables condiciones para cumplir funciones de orientación y asesoramiento respecto de la misma.

\section{CONCLUSIONES}

Para finalizar y a modo de concluir digamos que la realidad nos muestra que actualmente la relación biblioteca universitaria-usuario ha sufrido grandes cambios como consecuencia, entre otras cosas, del impacto que en ella han provocado las nuevas tecnologías de la información y de la comunicación.

El usuario tiene a su disposición los medios para acceder a la información prácticamente sin límites pero, creemos que en esta nueva situación se consolida efectivamente el rol mediador de la biblioteca universitaria apuntando a la formación de usuarios de la información críticos y selectivos.

Siguiendo a Estrada (8) "debemos señalar enfáticamente que la vida de las bibliotecas universitarias está asegurada porque es una institución de carácter único y eminentemente democrático que pone al alcance de todos, sin discriminación alguna, información 
sistematizada y actualizada constantemente, es una institución de servicio en la que predomina la difusión de la información".

El tema pasa, entonces, por el hecho de que la biblioteca universitaria tiene que plantearse nuevas formas de interacción con su comunidad de usuarios acordes a los momentos que estamos viviendo.

$\mathrm{Y}$ es con este objetivo que la presente ponencia intenta revalorizar la importancia de la biblioteca universitaria y la esencia de la función del profesional Bibliotecólogo que en la situación actual reasume nuevos perfiles de marcado protagonismo en un mundo de la información signado por el dinamismo y los continuos cambios.

\begin{abstract}
The relationship between the University Library and its users (students, teachers, researchers) has gone through great transformations as a consequence of the impact of new information technologies. The last trends indicate that the University Library is useless in a world in which information goes beyond the physical limits of institutions, where the user is in direct contact with the information through the computer. Despite this the University Library continues to play a fundamental role as an intermediate in the learning processes, as a research supporter and as a creator of new knowledge. One of the most appropriate ways to promote this role is establishing communication channels that strength the interactive connection library-user through the consolidation of users education. The librarian develops a fundamental role in this process. The current situation of Uruguayan University Libraries is described.
\end{abstract}

Keywords: university libraries/users/users education/

\title{
5 BIBLIOGRAFÍA CITADA
}

1) BARITE, Mario (1997). Reingeniería de las profesiones de la información y educación contínua : situación y prospectiva del caso uruguayo.- En: Trabajo presentado al Seminario Latinoamericano sobre Mercado y Nuevos Escenarios para el Profesional de la Información, Brasilia, IEL, 25-26 agosto, 1997.

2) DUARTE BARRIONUEVO, Miguel (1997). Perspectivas de la investigación en las bibliotecas universitarias.-En: Educ. y Bibl., 82:45-50, (1997).

3) QUESADA, Gabriela (1998). Gestión de calidad en bibliotecas : una aproximación al tema a partir de una encuesta realizada en Uruguay.- En: Informatio, no. (3/4):56-63, $(1998 / 1999)$.

4) TOCATLIAN, Jacques (1978). Formación de usuarios de la información : programas problemas y perspectivas.- En: Bol. Unesco, 32: 382-403, (1978). 
5) HERNÁNDEZ SALAZAR, Patricia, coord. (1997). En: Seminario Latinoamericano sobre formación de usuarios de la información y los estudios de usuarios.- México : UNAM, (1997).

6) DONNELLY, Kimberly (2000). Pondering their fledling years of teaching experience a group of faculty librarians talks about how they see themselves and how others now see them diferently.- En: Computers Libraries, 30(3):47-49, (2000).

7) CORDOBA, Saray (1996). Formación de usuarios para estudiantes universitarios: una experiencia con metodología participativa.- México: UNAM/CUIB, (1996).

8) ESTRADA CUZCAN, Alonso (1998). Bibliotecas universitarias : información, investigación, nuevas tecnologías. En: Curso Taller "Bibliotecas Universitarias : frente a los retos del siglo XXI", Universidad de Piura, Biblioteca Central, Perú, 16-17 octubre 1998.

\section{BIBLIOGRAFÍA CONSULTADA}

BEYA BONIFAZ, Matha (1993). Informe de asesoría para el establecimiento de la red de información bibliográfica de la Universidad de la República.- Montevideo : U.R., (1993).

BLÁQUEZ, Jesús (1996). Aplicaciones y usos de Internet en las bibliotecas y centros de documentación, el caso del Centro Washington Irving.- En: Ponencia presentada a "Internet World 1996" (1996).

CALVA GONZÁLEZ, Juan José (1996). Las necesidades de información de las comunidades científicas dentro del programa de formación de recursos humanos en servicios bibliotecarios y de información para la integración de la Red de Bibliotecas del Subsistema Humanidades y Ciencias Sociales.- En: Invest. Bibliot., México, 10(20):33-36, (1996).

COLAS, Alan (1999). La formation a l'usage de l'information dans l'enseignement supérieur.- En: Bull. Bibliot. France, 44(1):24-29, (1999).

FAVERO KRZYZANOWSKI, Rosaly; DE MORAIS IMPERATRIZ, Inés María (1998). Cooperacao e compartilhamento para o aperfeicoamento dos servicos bibliotecarios em bibliotecas universitarias.- En: Transinformacao, 10(1).15-32, janeiro/abril, (1998).

MAQUIÑA SILVA, Luis Alberto (1998). El impacto de las nuevas tecnologías en la biblioteca universitaria.- En: Curso Taller "Bibliotecas Universitarias : frente a los retos del siglo XXI", Universidad de Piura, Biblioteca Central, Perú, 16-17 octubre 1998.

THOMPSON, James; CARR, Reg (1990). La biblioteca universitaria: introducción a su gestión.- Madrid : Fundación Germán Sánchez Ruipérez, (1990). 